\title{
Invited Discussion on: Closed Rhinoplasty with a Mushroom-Shaped Costal Cartilage Graft in East Asian Noses
}

\author{
Nazım Cerkes ${ }^{1}$ (D)
}

Received: 10 December 2019/Accepted: 18 December 2019/Published online: 2 January 2020

(C) Springer Science+Business Media, LLC, part of Springer Nature and International Society of Aesthetic Plastic Surgery 2020

Level of Evidence $V$ This journal requires that authors assign a level of evidence to each article. For a full description of these Evidence-Based Medicine ratings, please refer to the Table of Contents or the online Instructions to Authors www.springer.com/00266.

The usual characteristics of East Asian noses are a low nasal dorsum, a short nose and a under projected nasal tip. In addition, East Asian alar cartilages are typically underdeveloped and the nasal tip skin is thick. In these patients, the goals of rhinoplasty surgery are to increase the nasal tip projection, elongate the nose and augment the nasal dorsum.

In the under projected nasal tip, there are various methods to increase tip projection and improve tip definition. The columellar strut graft is the most commonly used graft to increase tip projection. It strengthens the existing tip support, provides stability and increases tip projection when the medial crura are advanced on it. In cases that need a significant increase in the tip projection, lateral crural steal (advancing the lateral crura medially with spanning sutures) and the simultaneous use of a long and strong columellar strut give consistent results [1].

The other commonly used method to increase tip projection and adjust the tip position is fixation of the medial crura to a septal extension graft. The caudal septal extension graft is a rectangular cartilage that is extended off of the caudal septum. It is a useful graft to set projection and position of the nasal tip and elongate a short nose [2]. To maximize stability and avoid asymmetries, the caudal septal extension graft should be stabilized to the existing

Nazım Cerkes

dr@nazimcerkes.com

1 Cosmed Plastic Surgery Center, Hakki Yeten Cad. No 17/6, Ascioglu Plaza, Sisli, 34365 Istanbul, Turkey caudal septum with bilateral spreader grafts. Then the medial crura are fixed to the caudal margin of the septal extension graft with "tongue in groove" sutures to achieve the desired tip projection and rotation.

Patients that present with under projected tips most often have underdeveloped alar cartilages and ill-defined nasal tip contours. In these cases with underdeveloped lateral and medial alar cartilages, the overall length of the alar cartilage is short. In such cases, elongating the three legs of the nasal tip tripod with cartilage grafts increases tip projection and strengthens the tip while preventing upward rotation of nasal tip. In this method, the lower leg of the tripod (medial crura) is elongated using lateral crural steal in combination with a columellar strut or caudal septal extension graft. For elongation of the upper legs of the tripod, the lateral crura are first divided from the accessory cartilages, and then a lateral crural graft is placed between the lateral crura and the accessory cartilage to bridge the gap and reconstitute a stable nasal tip tripod [3].

After using one of these methods, if an additional increase in tip projection and further refinement is needed, a tip graft can be placed to increase tip projection and improve the tip contour. However, if the medial crural support is not sufficient, the use of tip grafts in isolation to increase tip projection may not be effective.

To set tip projection and position in Asian noses, one of the methods mentioned above or combination can be used. In East Asian patients, caudal septal extension graft stabilized with spreader grafts and/or elongation of legs of the nasal tip tripod can be very effective techniques for increasing tip projection and elongation of the nose. However, these methods require straight and strong cartilage struts. In the majority of primary Caucasian patients, sufficient septum cartilage can be harvested without weakening the septum cartilage. But in the East Asian nose, the 
septal cartilage is usually weak and small. For a satisfactory autologous reconstruction, including nasal length, increasing tip projection and augmenting, the low dorsum requires a significant amount of cartilage. Therefore, reconstruction with rib cartilage is the gold standard in East Asian noses. Due to the technical difficulty and long operation time when using autologous rib cartilage reconstruction, the most commonly used method in East Asian noses is placement of alloplastic implants which has a risk of extrusion in long term.

The method described in this article seems a useful solution in East Asian rhinoplasty patients. In East Asian patients with thick skin and a under projected tip, strong structural grafts prepared from rib will give better tip definition and a more predictable outcome. The best technique to elongate a short nose is a septal extension graft stabilized on the midline with bilateral spreader grafts [2]. The "mushroom graft" is essentially a strong columellar strut in combination with a tip onlay graft in one piece to increase tip projection. In this surgical technique, since the mushroom graft is extended off the caudal septum with bilateral spreader grafts, it works as a septal extension graft which elongates the nose.

In standard application of the septal extension graft to increase the tip projection, the medial crura are advanced on the septal extension graft in a more anterior position and in certain cases in combination with lateral crural steal in order to elongate the medial crura. But this may cause undesirable effects such as over-shortening of the lateral crura, up-rotation of the tip and widening of the columellalabial angle. The method described in this article may minimize the possible problems of advancing the medial crura on the septal extension graft because overly anterior advancement of the medial crura for increased tip projection is avoided and the increase in tip projection is achieved primarily from the cap portion of the "mushroom graft."

Although I have never used this method, there may be possible drawbacks of this method. In the manuscript, the author splits the lower half of the mushroom graft and places it on top of the anterior nasal spine and with the aim to keep it on the midline. However, it is not clearly explained in the text (and not demonstrated in the technical video clip) how the graft is stabilized on the midline to the anterior nasal spine with fixation or not. Although the video clip is not clear, it appears that the mushroom graft is sitting on the maxillary bone caudal to the ANS. One of the problems of septal extension grafts prepared from rib cartilage is shifting from the midline due to overlapping with ANS and/or the posterior part of the caudal septum. In these cases, the tip will be deviated from the midline and the patient will experience clicking due to movement of the graft over ANS during animation. Another problem can be tethering of the upper lip while smiling. However, if the septal extension graft is placed and secured properly anterior to the ANS, these problems will not occur.

In cases with an under projected tip due to short alar cartilages, I prefer to elongate the three legs of the nasal tip tripod with a strong columellar strut and lateral crural strut grafts for an anatomic structural reconstruction to increase nasal tip projection [3]. The method prevents upward rotation of the tip and gives extra projection. Using this method, an anatomic tip tripod can be achieved together with two tip defining points. A mushroom-shaped graft as demonstrated in the article will end up with a round tip or a pointed tip instead of resulting in an elegant, anatomic two tip defining points. The mushroom shape acts as an onlay tip graft and may have visibility problems in the long term. The follow-up period of the patients in the article is a maximum of 21 months, on average 15 months which is not sufficient to see long-term problems.

The authors use solid onlay grafts from the rib for dorsal augmentation in the cases presented in the manuscript. Long-term problems of the rib solid onlay grafts are warping, lack of adaptation and non-unification with the recipient bed. Instead of a solid onlay graft, a diced cartilage graft wrapped with rectus abdominis fascia could be a safer and easier solution for dorsal augmentation [4].

Due to concerns for the risk of scar development of the columellar scar, the authors used a closed approach. I believe that this technique can be performed more easily and accurately with an open approach. In my experience, a properly placed mid-columellar inverted $\mathrm{V}$ incision will heal well without a visible scar even in Fitzpatrick type V skin types.

However, despite the possible drawbacks, the "mushroom graft" together with bilateral septal extension grafts seems to be a useful technique for East Asian patients which can be performed using the close approach.

\section{Compliance with Ethical Standards}

Conflict of interest The author declares he has no conflict of interest to disclose.

Statement of Human and Animal Rights This article does not contain any studies with human participants or animals performed by the author.

Informed Consent For this type of study, informed consent is not required.

\section{References}

1. Guyuron B, Varghai A (2003) Lengthening the nose with a tongueand-groove technique. Plast Reconstr Surg 111:1533-1539

2. Cerkes N (2014) Structural grafting of the nasal tip. In: Rohrich RJ, Adams WP Jr, Ahmad J (eds) Dallas rhinoplasty nasal surgery by the masters, 3rd edn. CRC Press Inc., Boca Raton 
3. Cerkes N (2016) Nasal tip deficiency. Clin Plast Surg 43(1): $135-150$

4. Cerkes N (2016) Diced cartilage rectus abdominis fascia graft for dorsal augmentation. Plast Reconstr Surg 137:43-51
Publisher's Note Springer Nature remains neutral with regard to jurisdictional claims in published maps and institutional affiliations. 